\title{
CONTRIBUIÇÕES À SAÚDE MENTAL DO IDOSO NA ATENÇÃO PRIMÁRIA À SAÚDE: UMA REVISÃO INTEGRATIVA
}

\author{
Aline Pereira de Souza ${ }^{1}$, Kátia Terezinha Alves Rezende ${ }^{2}$, Maria José Sanches Marin ${ }^{3}$ \\ Silvia Franco da Rocha Tonhom ${ }^{4}$ e Daniela Garcia Damaceno ${ }^{5}$ \\ 1,2,3,4 Faculdade de Medicina de Marilia (Famema), Brasil, ${ }^{1}$ aline.psouza_enfer@hotmail.com \\ ${ }^{2}$ katialvesrezende@gmail.com, ${ }^{3}$ marnadia@terra.com, ${ }^{4}$ siltonhom@gmail.com \\ ${ }^{5}$ Universidade do Oeste Paulista, Brasil, daniela.garcia22@yahoo.com.br
}

\begin{abstract}
Resumo. O número da população idosa cresceu de maneira expressiva, identifica-se entre os idosos um aumento dos problemas de saúde mental, o que requer alerta. Este estudo teve como objetivo identificar as ações de promoção à saúde mental da pessoa idosa na atenção primária à saúde. As bases de dados para busca foram: Lilacs, Scopus, IBECS, Medline, CINAHL, BDENF e Index Psicologia utilizando-se os descritores "idoso", "Promoção da Saúde", "saúde mental" e "Atenção Primária a Saúde". A amostra final constituiu-se por 10 artigos. A análise de dados foi realizada por meio da análise de conteúdo, modalidade temática. Apresenta-se, nesse momento, uma temática, a saber, as potencias para efetivação da atenção psicossocial. Depreende-se que muitos avanços ainda são necessários ao tensionamento de ações na perspectiva psicossocial no contexto da atenção primária à saúde.
\end{abstract}

Palavras-chave: Idoso; Promoção da Saúde; Saúde Mental e Atenção Primária à Saúde.

\section{CONTRIBUTIONS TO THE MENTAL HEALTH OF THE ELDERLY IN PRIMARY HEALTH CARE: AN INTEGRATIVE REVIEW}

\begin{abstract}
The number of the elderly population has grown significantly, an increase in mental health problems is identified among the elderly, which requires alertness. This study aimed to identify actions to promote mental health in the elderly in primary health care. The databases for the search were: Lilacs, Scopus, IBECS, Medline, CINAHL, BDENF ande Psychology Index using the descriptors "elderly", "Health Promotion", "Mental Health" and "Primary Health Care". The final sample consisted of 10 articles. Data analysis was performed through content analysis, thematic modality. At this moment, a theme is presented, namely, the potential for effective psychosocial care. It appears that many advances are still needed to tension actions from a psychosocial perspective in the context of primary health care.
\end{abstract}

Keywords: Elderly; Health Promotion; Mental Health and Primary Health Care.

\section{INTRODUÇÃO}

O número da população idosa cresceu de maneira expressiva, atingindo um patamar de 13,5\% do total da população brasileira em 2018 (IBGE, 2018). Revela-se a necessidade de aperfeiçoar as políticas públicas existentes e ampliar à capacidade de assistir com qualidade essas pessoas. A atenção à saúde do idoso apresenta necessidades específicas caracterizadas pela sua cronicidade e complexidade, o que interfere na qualidade de vida, portanto requer alerta. Entre os idosos, identifica-se um aumento dos problemas de saúde mental; atribui-se aos eventos estressantes, presença de doenças, incapacidades e isolamento social. Além disso, representações sociais dos profissionais de saúde e da 
comunidade colaboram para que problemas relacionados à saúde mental dos idosos sejam confundidos com o processo natural do envelhecimento, prejudicando no diagnóstico e no tratamento (Onofri Júnior, Martins \& Marin, 2016).

O Plano Internacional de Ação sobre o Envelhecimento (PIAE), no que tange à saúde mental da pessoa idosa, prevê a realização de estratégias que favoreçam a prevenção dos sofrimentos mentais, a identificação precoce e o tratamento. Este visa desenvolver ações de educação e conscientização da população, a fim de atingir o envelhecimento saudável, fortalecer a rede de cuidados e apoio aos idosos envolvendo a família e a comunidade (Leandro-França \& Murta, 2014).

A Atenção Primária à Saúde (APS) é ordenadora do cuidado ao sistema de saúde e responsável pela resolução de grande parte das necessidades, espera-se assim, que a saúde do idoso seja assistida em suas múltiplas dimensões. Nesse sentido, o Núcleo Ampliado de Saúde da Família e Atenção Básica (NASF-AB), opera como equipe multiprofissional e interdisciplinar de intervenção integrada às equipes da APS; agrega-se às equipes, profissionais do campo de saúde mental (BRASIL, 2017). Os Caps (Centro de Atenção Psicossocial), sendo serviços de referência em saúde mental, também devem operar na lógica do apoio matricial às equipes da APS. No entanto, reconhece-se o desafio de fortalecê-los na prática dos serviços (Lima \& Dimenstein, 2016).

Salienta-se que os paradigmas sociais e a não aceitação da família ou da própria pessoa em sofrimento psíquico, são elementos que dificultam a prática do cuidado orientado à saúde mental dos idosos. Acresce-se a isso, preconceitos relacionados ao processo de envelhecimento, entre os próprios idosos. Constata-se ainda persistente a estigmatização acerca da "loucura" pela sociedade geral, como também entre os profissionais de saúde (Penido, 2013). A atenção à Saúde Mental do idoso na APS configura-se por práticas de cuidado ambulatoriais e pela presença de uma transição conceitual na visão acerca do processo de envelhecimento e Saúde Mental (Garcia, Moreira \& Oliveira, 2017). Apesar das experiências exitosas com a implantação da ESF, a visão centrada na doença persiste, principalmente em relação à população idosa, prevalecendo o modelo biomédico de atenção à saúde (Castro, Vidal, Saraiva, Arnaldo, Borges \& Almeida, 2018).

Frente a este contexto, acredita-se na importância de se transpor os desafios da produção do cuidado em saúde mental e potencializar o cuidado territorializado, considerando a necessidade emergente de avançar na promoção da saúde e prevenção de agravos à 
saúde mental dos idosos. Questiona-se: quais as ações de promoção à saúde mental da pessoa idosa no contexto da APS disponível na literatura?

\section{OBJETIVO}

O presente estudo tem por objetivo identificar as ações de promoção e prevenção à saúde mental da pessoa idosa no contexto da atenção primária à saúde disponível na literatura.

\section{METODOLOGIA}

Trata-se de uma revisão integrativa. Este tipo de estudo corresponde a um método de pesquisa que viabiliza análise de pesquisas científicas de modo sistemático e amplo, favorecendo a caracterização e divulgação do conhecimento produzido (Botelho, Cunha \& Macedo, 2011). Destaca-se pela exigência dos mesmos padrões de rigor, clareza e replicação utilizados em estudos primários. Além de constituir-se a mais ampla abordagem metodológica referente às revisões de literatura (Cerqueira, Cardoso, Viana, Lopes, 2018). A revisão integrativa consiste no cumprimento de 6 etapas: 1) identificação do tema e seleção da questão de pesquisa; 2) estabelecimento dos critérios de elegibilidade; 3) identificação dos estudos nas bases científicas; 4) avaliação dos estudos selecionados e análise crítica; 5) categorização dos estudos; 6) avaliação e interpretação dos resultados e apresentação dos dados na estrutura da revisão integrativa (Botelho, Cunha \& Macedo, 2011).

O estudo foi norteado por protocolo elaborado pelas pesquisadoras, que possibilitou a definição clara dos propósitos da investigação e a identificação das variáveis de interesse facilitando a seleção da amostra, a manutenção do foco e limites para a investigação.

A questão de pesquisa foi elaborada de acordo com a estratégia PICo (P-população; Iinteresse; Co - Contexto) (Lockwood, et al., 2017). Considerando-se a seguinte estrutura: P - pessoas idosas; I - ações de promoção de saúde mental; e Co- atenção básica. Dessa forma, esta investigação foi conduzida a partir da seguinte questão: Quais as ações de promoção e prevenção de saúde mental da pessoa idosa no contexto da atenção primária a saúde disponível na literatura?

O levantamento bibliográfico foi realizado julho de 2019 nas bases eletronicas de dados: US National Library of Medicine (PUMED); Literatura Latino-Americana e do Caribe em Ciências da Saúde (LILACS); Cumulative Index to Nursing and Allied Health Literature (CINAHLEbsco); Web of Science e Scopus (Elsevier); BDENF - Enfermagem; IBECS e Index 
Psicologia - Periódicos técnico-científicos. Para a busca nas bases de dados foram selecionados descritores presentes nos Descritores em Ciências da Saúde (DeCS) e seus equivalentes no idioma inglês no Medical Subject Headings (MeSH) e List of Headings do CINAHL.

Adotaram-se como critérios de inclusão foram: artigos primários que apresentavam de promoção em saúde na atenção primária, publicados no período de 2014 a 2019, nos idiomas inglês, português ou espanhol e com texto completo disponível. Os critérios de exclusão foram: editorais, teses, dissertações, artigos de revisão, àqueles já selecionados na busca em outra base de dados e que não respondiam à questão da pesquisa.

Para sistematizar a coleta utilizou-se formulário de busca avançada respeitando as peculiaridades de cada base de dados. Assim, foi utilizada a combinação boleana: idoso $O R$ aged OR elderly OR geriatric OR geriatrics AND Promoção da Saúde OR Health Promotion OR Educação Paciente OR Patient Education OR Health Education OR Educação em Saúde AND saúde mental OR Mental Health AND Primary Health Care OR Atenção Primária Saúde OR Atenção Basica OR Estrategia Saúde da Família.

A seleção dos artigos foi realizada de forma cega e independente por duas revisoras, que após leitura de títulos e resumos obtiveram índice de concordância superior a $90 \%$. No intuito de assegurar a busca ampla, os manuscritos foram acessados por meio do portal de periódicos da Coordenação de Aperfeiçoamento de Pessoal de Nível Superior (CAPES), em Virtual Private Network (VPN) disponibilizada pela Universidade Estadual Paulista "Júlio de Mesquita Filho".

Para a extração e síntese dos artigos selecionados, utilizou-se de instrumento construído pelas próprias investigadoras. Foram extraídos os dados: autor, ano, pais da publicação, tipo de estudo, ações e contribuições. Quanto ao nível de evidência, os artigos foram avaliados segundo o conceito proposto pela Prática Baseada em Evidências, classificadas em sete níveis (Silva, Pontes, Sousa \& Vasconcelos, 2019).

Segundo a literatura adotada, é considerado nível de evidencia 1, evidências provenientes de revisão sistemática ou metanálise de ensaios clínicos randomizados controlados ou de diretrizes clínicas baseadas em revisões sistemáticas de ensaios clínicos randomizados controlados; nível 2, evidências derivadas ensaio clínico randomizado controlado bem delineado; nível 3, evidências ensaios clínicos bem delineados sem randomização; nível 4, 
evidências estudos de coorte e de caso-controle bem delineados; nível 5, evidências de revisão sistemática de estudos descritivos e qualitativos; nível 6, evidências de um único estudo descritivo ou qualitativo; nível 7, evidências de opinião de autoridades e/ou relatório de especialistas.

A coleta de dados foi realizada em duas etapas. A primeira consistiu em identificar 3.403 publicações, dos quais, após aplicação dos critérios de inclusão e exclusão, foram selecionados para a amostra desta revisão 10 artigos. Para a seleção das publicações, foram seguidas as recomendações do Preferred Reporting Itens for Systematic Reviews anda Meta-Analyses (PRISMA), conforme apresentado na figura 1:

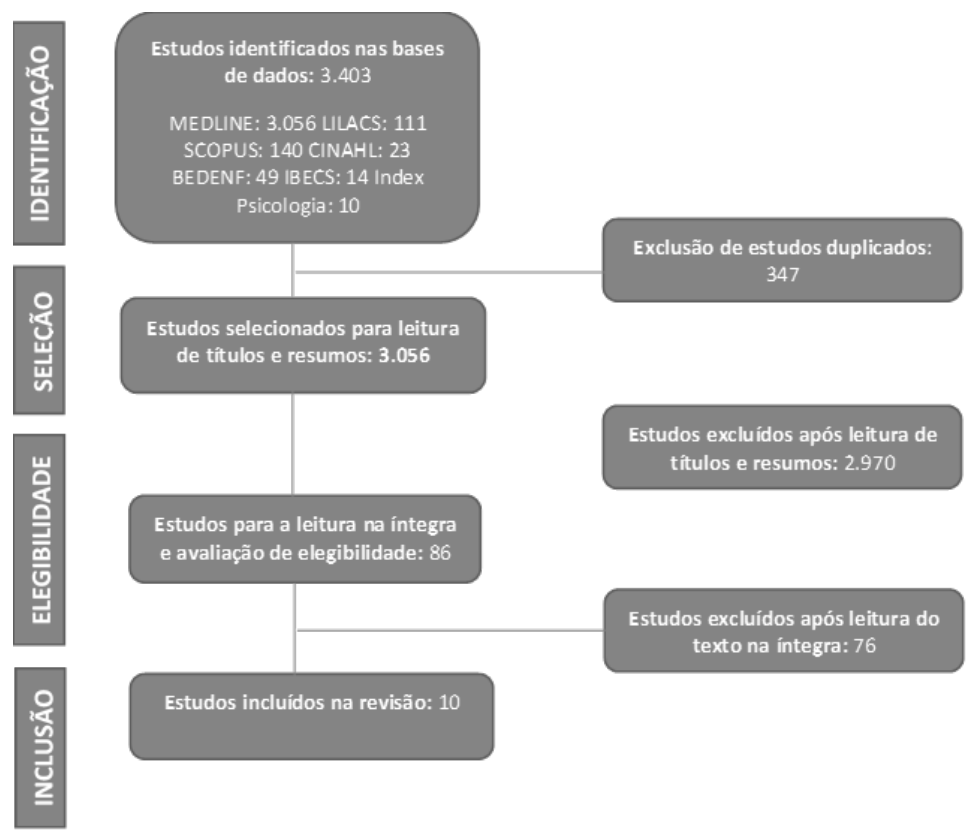

Figura 1 - Fluxograma de seleção dos estudos primários, elaborado a partir da recomendação PRISMA. Marília, SP, Brasil, 2019

Os dados da foram tabulados em forma de quadro e a análise crítica e síntese reflexiva foram realizadas de forma descritiva segundo os achados da efetivação da atenção psicossocial no contexto da atenção primária.

Por tratar-se de revisão integrativa, a investigação não foi submetida a Comitê de Ética em Pesquisa, contudo foram preservadas as concepções dos autores dos manuscritos utilizados na presente investigação. 


\section{RESULTADOS E DISCUSSÃO}

\subsection{Caracterização dos artigos}

Dos 10 artigos incluídos na revisão integrativa (Quadro 1), a maioria foi publicada entre os anos de 2017 e 2019; quatro em periódicos do Brasil; um do Reino Unido; dois dos EUA; dois da Austrália e um do Chile. Em relação à classificação hierárquica metodológica proposta pela Prática Baseada em Evidências em sete níveis (Silva, Pontes, Sousa \& Vasconcelos, 2019) foram identificados: um artigo no nível dois (ensaio clínico randomizado); um no nível três (ensaio clínico sem randomização); um no nível quatro (estudos de coorte ou caso-controle) cinco no nível seis (estudo descritivo ou qualitativo) e dois no nível sete (opinião de autoridades ou relatório de comitê). Constata-se, portanto, estudos sobre a temática em diferentes países do mundo, entretanto com grau de evidência baixo, conforme mostra o Quadro 1.

Quadro 1 - Síntese da análise dos artigos selecionados. Marília- SP. 2020

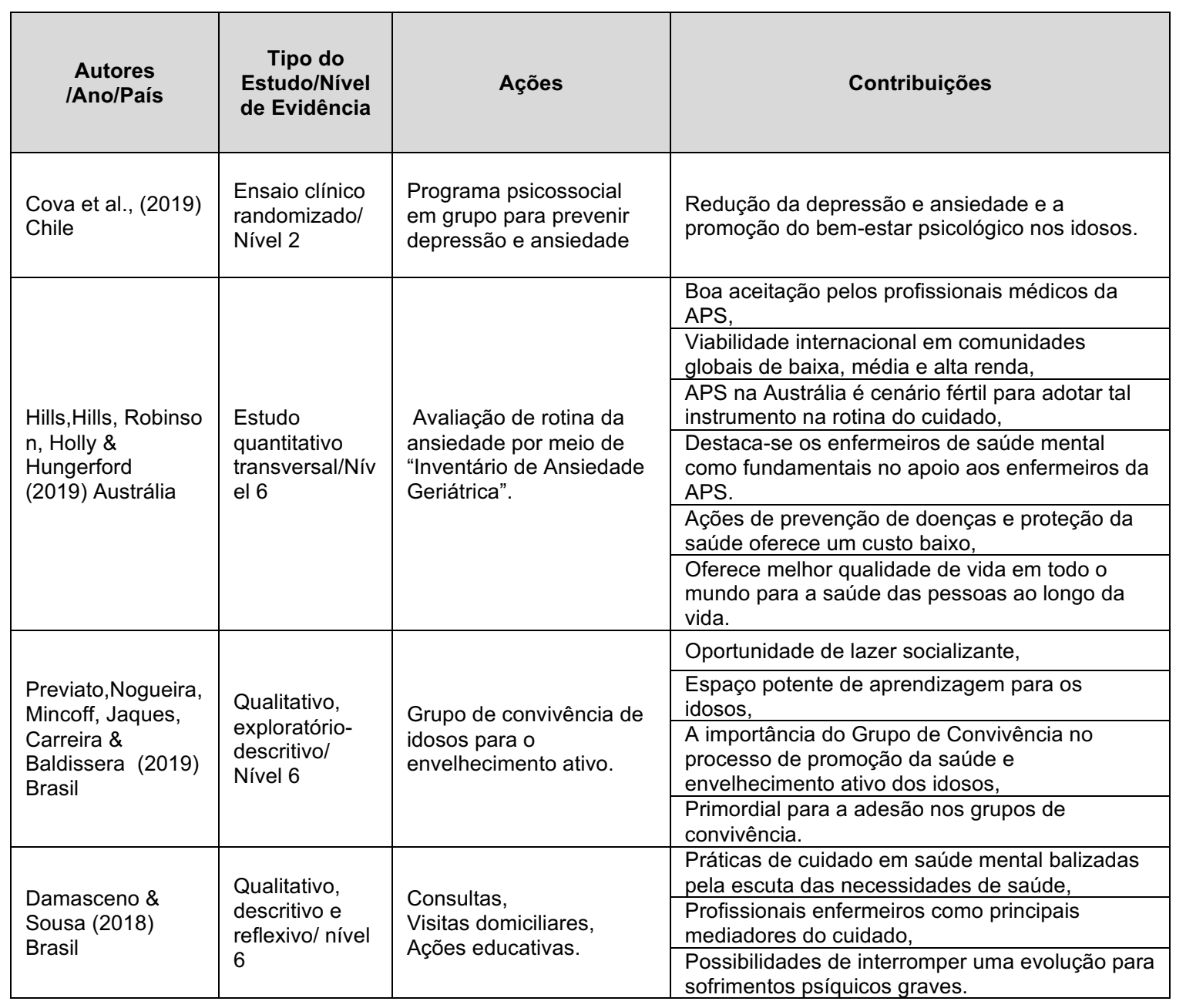




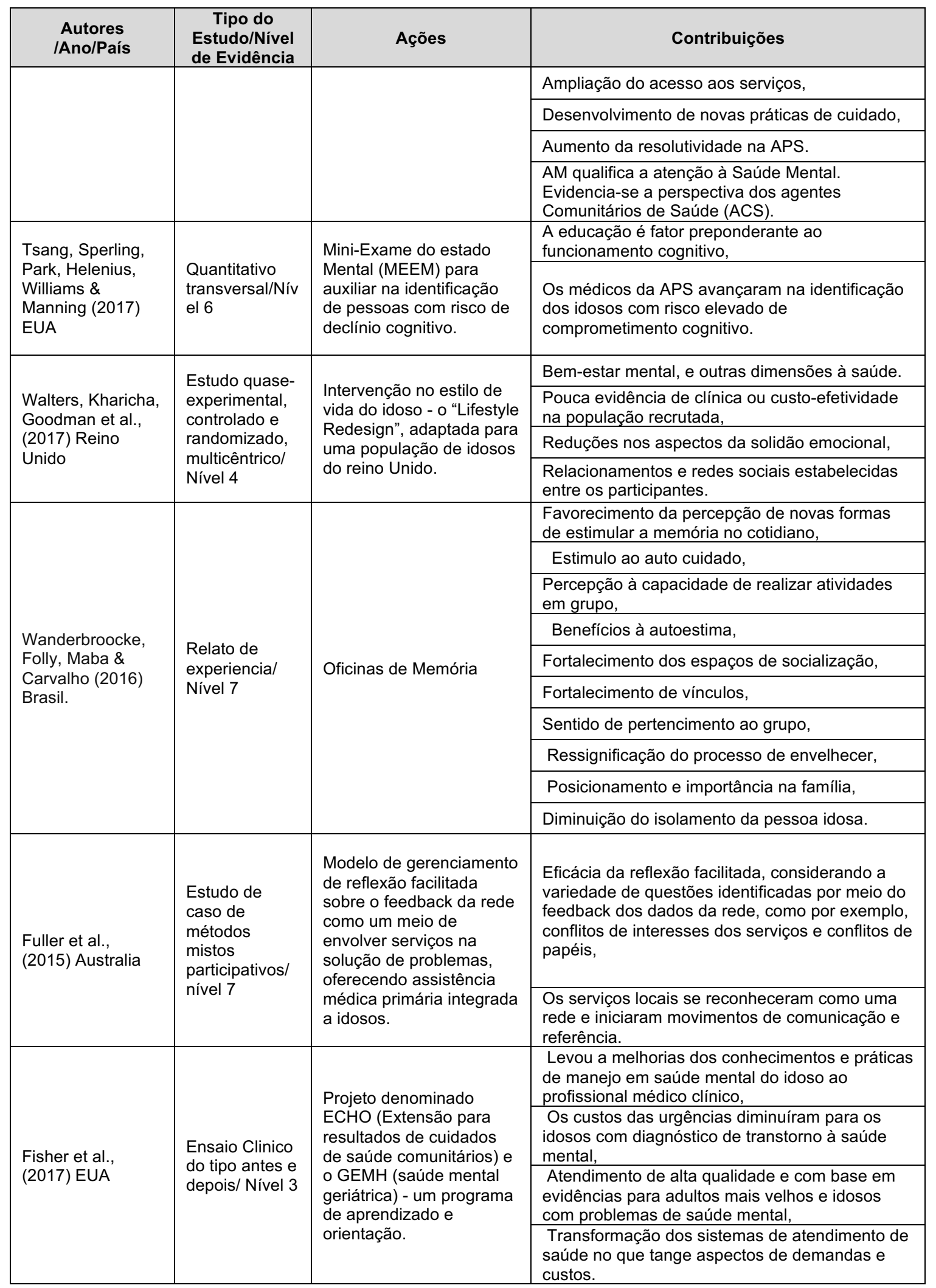




\subsubsection{Ações de promoção e prevenção à saúde mental da pessoa idosa no contexto da atenção primária}

De modo geral, considerando a produção de saúde mental orientada à atenção psicossocial; identifica-se na revisão integrativa contribuições nessa perspectiva no contexto da APS. Evidencia-se que intervenções voltadas à prevenção, operacionalizadas em grupos reduzem sintomas depressivos e proporciona melhor qualidade psicológica aos idosos. Considera-se potente abordagens no cuidado em grupo, com centralidade na promoção da saúde e saúde mental na APS. Ressalta-se que estratégias nessa direção, valorizam a essência humana, promove escuta, compreensão, fortalecimento de vínculos e laços afetivos entre as pessoas da comunidade e os profissionais (Cova, et al., 2019).

Dentre as estratégias psicossociais, encontram-se os Grupo de Convivência de Idosos. Caracteriza-se por atividades de lazer desenvolvidas em grupo, sendo estas, brincadeiras, danças, atividades manuais e passeios externos, que produzem satisfação dos participantes do grupo, decorrentes principalmente dos afetos gerados nesse processo. Denota-se ainda, a autonomia na escolha do que querem aprender, com foco no protagonismo dos idosos, acrescenta-se, que participar do grupo nos momentos de lazer, favorece a socialização, o encontro e interação com diferentes pessoas, estreitamento de vínculos, rompendo com o isolamento social (Previato, Nogueira, Mincoff, Jaques, Carreira \& Baldissera 2019).

Revelou-se a importância do Grupo de Convivência no processo de promoção da saúde e envelhecimento ativo dos idosos, que atribuem melhoras a saúde global. Nesse sentido, evidencia-se que a promoção da saúde é fundamental à qualidade de vida e determinantes comportamentais da população idosa, com vista ao envelhecimento ativo. Apontam para a potência desses espaços como dispositivo de desinstitucionalização, favorecendo o desenvolvimento psicossocial (Previato, Nogueira, Mincoff, Jaques, Carreira \& Baldissera 2019).

Outra estratégia encontrada foi a Oficina de Memória (OM) que se estabeleceu em três categorias, a saber: achar que já estava apresentando falhas na memória, medo de perder a memória, desejo de participar de atividades de promoção à saúde. Destaca-se o medo de perder a memória e o desejo de participação nas atividades de promoção à saúde (Wanderbroocke et al., 2016). Revela-se os benefícios à autoestima a partir das OM, e o fortalecimento dos espaços de socialização, onde falavam sobre suas vivências, angústias, favorecendo trocas e estreitando vínculos, o que contribuiu para dar sentido de 
pertencimento ao grupo, à ressignificação do processo de envelhecer, de sua posição e importância na família. Os idosos sugerem que a OM deveria ser um trabalho contínuo oferecido pela UBS. Reforça-se a potência da APS e seu amplo escopo de atuação, considerando as necessidades do território (Wanderbroocke et al., 2016). Ainda na perspectiva de socialização, aponta-se reduções significativas nos aspectos da solidão emocional para os idosos que participaram da intervenção Lifestyle Matters, sendo esta, uma intervenção preventiva com vista a promoção à saúde mental, envolvendo sessões semanais em grupo, com intervenções no estilo de vida do idoso pautada na ocupação, a fim de promover maior interação social (Walters et al., 2017).

Emergiu-se nesta revisão, o apoio matricial (AM) como ferramenta indispensável no cuidado em saúde mental na APS. Destaca-se a necessidade de integração da saúde mental ao cotidiano das práticas da APS para a efetivação da integralidade do cuidado em saúde, tendo o matriciamento em saúde mental como uma importante estratégia para efetivação dessa articulação (Amaral et al., 2018). Nesse sentido, os enfermeiros especialistas em saúde mental, foram apontados como fundamentais no apoio aos enfermeiros da APS na implementação de uma avaliação de rotina da ansiedade em idosos na Australia. Trata-se de estratégias de ação de proteção e prevenção de riscos e agravos, tendo o enfermeiro especialista em saúde mental, orientado na perspectiva de matriciamento, responsável por qualificar os profissionais da APS de forma a reconhecer e responder às necessidades de saúde mental nas pessoas idosas na direção de prevenção às doenças e promoção da atenção psicossocial (Hills et al., 2019).

Identifica-se efeitos do AM em quatro dimensões, sendo estas: mudanças na atitude dos profissionais; ampliação do acesso aos serviços; desenvolvimento de novas práticas de cuidado e aumento da resolutividade. Enfatiza-se a capacidade de empatia e compreensão referente à pessoa em sofrimento psíquico. Salienta-se as relações e a criatividade para produção do cuidado, bem como, a busca ativa realizada pelos ACS, o estreitamento de vínculo das pessoas envolvidas, favorecendo a potencialidade das tecnologias leves, leveduras e duras (Merhy, 2005; Amaral et al., 2018).

A versatilidade nas possibilidades de cuidado em saúde mental produzidas pelos ACS, haja visto o caráter heterogêneo desses profissionais e, da integração de competências técnicas e conhecimentos advindos de experiencias prévias, fortalece ações significativas, sem o predomínio do saber e da pratica biomédica (Amaral, et al., 2018) Aponta-se, ainda, que o 
AM trouxe aos ACS um novo sentido referente ao CAPS, pois havia certo desconhecimento anterior acerca da existência do serviço, viabilizou-se a construção de instrumentos de comunicação para direcionamento dos casos, com vista ao estabelecimento de uma integração mais efetiva entre CAPS e ESF (Amaral et al., 2018).

Outra intervenção, foi o programa de Telementoring como o Project ECHO GEMH, que levou a melhorias dos conhecimentos e práticas de manejo em saúde mental do idoso ao profissional médico clínico da APS. Infere-se que os custos das urgências diminuíram para os idosos com diagnóstico de transtorno à saúde mental (Fisher, 2017). Reforça-se que desenvolver ações de prevenção de doenças e promoção da saúde oferece um custo baixo, acrescenta-se, o potencial de oferecer melhor qualidade de vida em todo o mundo para a saúde das pessoas ao longo da vida (HILLS et al., 2019). Revela-se a eficácia da reflexão facilitada por meio do feedback da rede, no qual emergiram-se uma variedade de questões, como por exemplo, conflitos de interesses dos serviços e conflitos de papéis. Nesse sentido, as discussões ajudaram os serviços locais a se identificarem como uma rede e a iniciar movimentos de comunicação e referência para solução de problemas (Fuller et al.; 2015).

\section{CONCLUSÕES}

Considera-se que o estudo alcançou o seu objetivo, pois por meio dele identificou-se as ações de promoção à saúde mental da pessoa idosa no contexto da APS disponível na literatura. Visando garantir a atenção psicossocial aos idosos em sofrimento psíquico, identifica-se que o momento é de ampliação do escopo de ações oferecidas a essa população. Dessa forma, evidencia-se esforços para a produção do cuidado na direção da integralidade vislumbrando a atenção psicossocial. Revela-se o apoio matricial como ferramenta indispensável no cuidado psicossocial, que se firma como recurso de construção de novas práticas em saúde mental, contudo, reconhece-se o desafio de fortalecê-los na prática dos serviços.

Assim, percebe-se que o método escolhido, a revisão integrativa, possibilitou identificar que, embora haja um movimento para a ampliação das ações na lógica da promoção da saúde mental na APS, este mostra-se incipiente ao tensionamento de ações na perspectiva psicossocial. Desse modo, o método revelou-se potente para a compreensão da temática.

Pode-se identificar como limitante da pesquisa o número reduzido de artigos selecionados, contudo, o material identificado possibilitou construir um panorama, nacional e internacional, 
sobre as ações de prevenção à doença e promoção a saúde da pessoa idosa na atenção primária.

\section{REFERÊNCIAS}

IBGE - INSTITUTO BRASILEIRO DE GEOGRAFIA E ESTATístICA. Censo demográfico: resultados preliminares - São Paulo. Rio de Janeiro, 2018.

Onofri Júnior, Venício Aurélio, Martins, Vinícius Spazzapan, \& Marin, Maria José Sanches. (2016). Elderly health care in the Family Health Strategy and the prevalence of common mental disorders. Revista Brasileira de Geriatria e Gerontologia, 19(1), 21-33. https://doi.org/10.1590/1809-9823.2016.15004

Leandro-França, Cristineide, \& Giardini Murta, Sheila. (2014). Prevenção e promoção da saúde mental no envelhecimento: conceitos e intervenções. Psicologia: Ciência e Profissão, 34(2), 318329. https://doi.org/10.1590/1982-3703001152013

BRASIL. Ministério da Saúde. Secretaria de Atenção à Saúde. Departamento de Atenção Básica. Cobertura da atenção básica. 2017 [acesso em 2019]. Disponível em: http://dab.saude.gov.br/portaldab/.

Lima, Maura e Dimenstein, Magda. (2016). O apoio matricial em saúde mental: uma ferramenta de apoio à atenção à crise. Interface - Comunicação, Saúde, Educação, 20 (58), 625-635. Epub 17 de maio de 2016. https://doi.org/10.1590/1807-57622015.0389

PENIDO C. Apoio matricial em saúde mental no contexto da saúde coletiva. In: Paulon S, Neves R, organizadores. Saúde Mental na Atenção Básica: a territorialização do cuidado. Porto Alegre: Sulina; 2013. p. 13-39.

Garcia, B., Moreira, D., \& Oliveira, P. (2017). Saúde Mental do Idoso na Atenção Primária: Uma Análise das Percepções de Profissionais de Saúde. Revista Kairós : Gerontologia, 20(4), 153-174. doi:https://doi.org/10.23925/2176-901X.2017v20i4p153-174

Castro, Ana Paula Ribeiro de, Vidal, Eglídia Carla Figueirêdo, Saraiva, Ana Raquel Bezerra, Arnaldo, Sofia de Moraes, Borges, Ana Maria Machado e Almeida, Maria Irismar de. (2018). Promoção da saúde do idoso: ações na atenção básica à saúde. Revista Brasileira de Geriatria e Gerontologia, 21 (2), 155163. https://doi.org/10.1590/1981-22562018021.170133

Botelho, L. L. R., Cunha, C. C. de A., \& Macedo, M. (2011). O MÉTODO DA REVISÃO INTEGRATIVA NOS $\begin{array}{llll}\text { ESTUDOS ORGANIZACIONAIS. Gestão } & \text { E } & \text { Sociedade, 5(11), } & \text { 121-136. }\end{array}$ https://doi.org/10.21171/ges.v5i11.1220

Cerqueira, Ana Carolina Dantas Rocha, Cardoso, Maria Vera Lúcia Moreira Leitão, Viana, Tamires Rebeca Forte, \& Lopes, Márcia Maria Coelho Oliveira. (2018). Revisão integrativa da literatura: sono em lactentes que frequentam creche. Revista Brasileira de Enfermagem, 71(2), 424-430. https://doi.org/10.1590/0034-7167$\underline{2016-0480}$

Lockwood C, Porrit K, Munn Z, Rittenmeyer L, Salmond S, Bjerrum M, Loveday H, Carrier J, Stannard D. Chapter 2: Systematic reviews of qualitative evidence. In: Aromataris E, Munn Z (Editors). Joanna Briggs Institute Reviewer's Manual. The Joanna Briggs Institute, 2017. Available from https://reviewersmanual.joannabriggs.org/

Silva, Naélia Vidal de Negreiros da, Pontes, Cleide Maria, Sousa, Nayara Francisca Cabral de, \& Vasconcelos, Maria Gorete Lucena de. (2019). Tecnologias em saúde e suas contribuições para a promoção do aleitamento materno: revisão integrativa da literatura. Ciência \& Saúde Coletiva, 24(2), 589602. https://doi.org/10.1590/1413-81232018242.03022017 
Minayo, M.C.S. O Desafio do Conhecimento: pesquisa qualitativa em saúde. São Paulo: Hucitec, 2013.

Cova, F., Rincón, P., Bustos, C., Streiner, D., King, M., Saldivia, S., ... Novoa, C. (2019). Randomized cluster trial of a parenting program in Chile: Key mediators in the decrease in behavior problems in preschool children. Clinical Child Psychology and Psychiatry, 135910451986412. doi:10.1177/1359104519864124

Hills, D., Hills, S., Robinson, T., \& Hungerford, C. (2019). Mental Health Nurses Supporting the Routine Assessment of Anxiety of Older People in Primary Care Settings: Insights from an Australian Study. Issues in Mental Health Nursing, 1-6. doi:10.1080/01612840.2018.1517285

Previato, GF, Nogueira, IS, Mincoff, RCL, Jaques, AE, Carreira, L. e Baldissera, VDA (2019). Grupo de convivência de idosos na atenção básica à saúde: contribuições para o envelhecimento ativo. Revista de Pesquisa: Care and Fundamental , 11 (1), 173-180. Recuperado de http://ciberindex.com/c/ps/P111173

Damasceno, V., \& Sousa, F. (2018). Atenção à saúde mental do idoso: a percepção do enfermeiro. Revista de Enfermagem da UFPE on line, 12 (10), 2710-2716. doi: https://doi.org/10.5205/1981-8963v12i10a234647p2710-2716-2018

Amaral, Carlos Eduardo Menezes, Torrenté, Mônica de Oliveira Nunes de, Torrenté, Maurice de, \& Moreira, Carolina Pinheiro. (2018). Apoio matricial em Saúde Mental na atenção básica: efeitos na compreensão e manejo por parte de agentes comunitários de saúde. Interface - Comunicação, Saúde, Educação, 22(66), 801-812. Epub 17 de maio de 2018.https://doi.org/10.1590/1807-57622017.0473

Tsang, S., Sperling, S. A., Park, M.-H., Helenius, I. M., Williams, I. C., \& Manning, C. (2017). Health Variables Are Informative in Screening for Mild Cognitive Impairment Among Elderly African Americans. Journal of Applied Gerontology, 073346481771196. doi:10.1177/0733464817711961

Walters, K., Kharicha, K., Goodman, C., Handley, M., Manthorpe, J., Cattan, M., ... Iliffe, S. (2017). Promoting independence, health and well-being for older people: a feasibility study of computer-aided health and social risk appraisal system in primary care. BMC Family Practice, 18(1). doi:10.1186/s12875-017-0620-6

Wanderbroocke, A., Folly, P., Maba, P., \& Carvalho, T. (2016). Oficina de memória para idosos em uma unidade básica de saúde: um relato de experiência. Psicologia Revista, 24(2), 253-263. Recuperado de https://revistas.pucsp.br/psicorevista/article/view/27798

Fuller, J., Oster, C., Muir Cochrane, E., Dawson, S., Lawn, S., Henderson, J., ... Reed, R. L. (2015). Testing a model of facilitated reflection on network feedback: a mixed method study on integration of rural mental healthcare services for older people. BMJ Open, 5(11), e008593-e008593. doi:10.1136/bmjopen-2015008593

Fisher, E., Hasselberg, M., Conwell, Y., Weiss, L., Padrón, N. A., Tiernan, E., ... Pagán, J. A. (2017). Telementoring Primary Care Clinicians to Improve Geriatric Mental Health Care. Population Health Management, 20(5), 342-347. doi:10.1089/pop.2016.0087 